partner, and condom use with them is significantly lower than with clients. Therefore, we can conclude that unprotected sex with non-commercial partners is the main risk factor to get STI for FSW in Armenia. These findings highlight the need for HIV prevention interventions that engage both FSW and their sex partners, especially non-commercial. Improvement of condom negotiation skills, provision of HIV/AIDS risk and transmission education should be the focus of interventions targeting FSW. Additionally, health care and other service providers should encourage routine HIV testing and STI screening for FSW and their partners.

\section{P3.16 IMPACT OF RAPID SUSCEPTIBILITY PROFILING ON THE EMERGENCE AND SPREAD OF ANTIBIOTIC RESISTANCE IN GONORRHOEA}

${ }^{1}$ Ashleigh Tuite, ${ }^{2}$ Katherine Hsu, ${ }^{3}$ Thomas L Gift, ${ }^{4}$ Joshua A Salomon, ${ }^{5}$ Yonatan H Grad. ${ }^{1}$ Havard University, Boston, USA; ${ }^{2}$ Massachusetts Department of Public Health, Boston, USA; ${ }^{3}$ Centres for Disease Control and Prevention, Atlanta, USA; ${ }^{4}$ Harvard Univeristy, Boston, USA; ${ }^{5}$ Harvard University, Boston, USA

\subsection{6/sextrans-2017-053264.253}

Introduction Increasing antibiotic resistance limits treatment for gonorrhoea. We examined the extent to which a hypothetical point-of-care (POC) test reporting antibiotic susceptibility profiles could delay emergence of resistance and prolong effectiveness of existing antibiotics.

Methods We developed a deterministic compartmental model describing gonorrhoea transmission in a risk-stratified singlesex population with three different antibiotics available to treat infections. Probabilities of resistance emergence on treatment and fitness costs associated with resistance were based on characteristics of fluoroquinolones, azithromycin, and ceftriaxone, as inferred from a previous phylogenomic analysis. We compared strategies in which a POC test was used to guide therapy in varying proportions of cases against the current empiric approach (dual treatment with azithromycin plus ceftriaxone).

Results Based on current estimates of gonoccoal susceptibility patterns in the United States, the model indicated that continued empiric dual antibiotic treatment without POC testing resulted in $>5 \%$ of isolates being resistant to both azithromycin and ceftriaxone within 15 years. When POC testing was used in $10 \%$ of identified cases, this time was delayed by 4 years, while time to reach a $1 \%$ prevalence of triply-resistant strains was delayed by 5 years. With POC testing in $>55 \%$ of identified cases, it took over 100 years for dual and triple resistance to exceed $1 \%$, and with POC testing in $\geq 75 \%$ of cases, strains resistant to azithromycin and/or ceftriaxone did not persist in the population. Results were sensitive to assumptions about fitness costs and test sensitivity only when POC test deployment was relatively low $(<25 \%)$.

Conclusion Rapid diagnostics that indicate antibiotic susceptibility have the potential to extend the usefulness of existing antibiotics for treatment of gonorrhoea. More broadly, integration of evidence on fitness costs associated with resistance can enhance strategies for rational antibiotic selection and further delay emergence of resistance.

\section{P3.17 QUALITY OF LIFE IN HIVIAIDS PATIENTS- AN EXPLORATION}

Asmin Sha. Al lqbal Hospital, Thrissur, India

\subsection{6/sextrans-2017-053264.254}

Introduction HIV/AIDS impacts heavily on the infected individual and the society at large, there is therefore a need to evaluate the quality of life of HIV-infected individuals.

Objectives: To assess the impact of HIV/AIDS on the Health related quality of life (HRQOL) of people living with HIV/AIDS (PLWHA), and to investigate the determinants of the QOL of PLWHA.

Methods A descriptive cross-sectional study design was used. One hundred and three (103) PLWHA accessing healthcare were consecutively selected. A questionnaire, containing data on socio-demographic and medical profiles, on the WHOQOL-HIV Bref was used to assess each study participant. HRQOL was evaluated to assess quality of life domains that included physical and physiological health, level of independence, social relationships, environment, and spirituality/religion/ personal beliefs. Means, standard deviations, and statistical tests for differences were performed.

Results The mean age of the respondents was 41.0 (range 21$73) ; 48(46.6 \%)$ of the participants were males. The QOL mean scores were highest for the spirituality/religion/personal beliefs domain $(16.88 \pm 2.83)$ and lowest for the environment domain (14.08 \pm 1.95$)$. The overall QOL mean scores in the other four domains were similar: physical health $(15.92$ $\pm 3.05)$, psychological health $(15.35 \pm 3.20)$, level of independence $(15.90 \pm 3.52)$, social relationships $(15.11 \pm 2.26)$. Significant differences were observed in all domains among respondents with family support compared to those without family support. Similarly, asymptomatic patients had significantly higher QOL scores compared to symptomatic patients. Improved QOL was influenced by higher educational levels in all domains except the spirituality/religion/personal beliefs domain.

Conclusion The impact of HIV on the HRQOL was highest in the environment and social relationships domains. Also, HIV serostatus, presence of family support, and educational levels had significant effects on the QOL of PLWHA.

\section{P3.18 MONITORING CHLAMYDIA TRACHOMATIS INFECTIONS AFTER TREATMENT FOR TEST OF CURE PURPOSES}

${ }^{1} \mathrm{~B}$ Versteeg, ${ }^{1,2} \mathrm{SM}$ Bruisten, ${ }^{1} \mathrm{~T}$ Heijman, ${ }^{1} \mathrm{~W}$ Vermeulen, ${ }^{1} \mathrm{MS}$ van Rooijen, ${ }^{1,4} \mathrm{AP}$ van Dam, ${ }^{1,2}$ MF Schim van der Loeff, ${ }^{1,2,3} \mathrm{HJC}$ de Vries, ${ }^{1,4} \mathrm{M}$ Scholing. ${ }^{1}$ Department of Infectious Diseases, Public Health Service Amsterdam, Amsterdam, the Netherlands; ${ }^{2}$ Centre for Infection and Immunity Amsterdam (CINIMA), Academic Medical Centre, University of Amsterdam, Amsterdam, the Netherlands; ${ }^{3}$ Department of Dermatology, Academic Medical Centre, University of Amsterdam; ${ }^{4}$ Department of Medical Microbiology, OLVG General Hospital, Amsterdam, The Netherlands

\subsection{6/sextrans-2017-053264.255}

Introduction Performing a test of cure (TOC) could demonstrate success or failure of antimicrobial treatment of C. trachomatis (CT) infection, but the value of using a nuclear acid amplification test (NAAT) based TOC after treatment is subject to discussion, as the presence of CT nucleic acids after treatment may be prolonged and intermittent without the presence of infectious bacteria. We used cell culture to assess if a NAAT positive TOC indicates the presence of viable CT. 
Methods We analysed follow up (FU) data from women with a CT infection who visited the STI clinic of Amsterdam, the Netherlands, from September 2015 through June 2016. After giving informed consent, participants underwent baseline and three FU speculum examinations to obtain cervical swabs for both CT culture and NAAT testing. Speculum examinations were scheduled at 7, 21 and 49 days after treatment (single dose $1000 \mathrm{mg}$ azithromycin). Collected samples were analysed using a RNA and DNA-based NAAT. CT cell culture was performed on all samples at baseline, and in FU samples that were NAAT-positive. Clearance was defined as conversion to negative NAAT results at any FU visit.

Results We included 78 women with NAAT proven CT infection prior to receiving treatment of whom 58 (74\%) were also culture positive. At the first visit after treatment (median 7 days; IQR 7-8) 44 (47\%) women were NAAT positive, of whom three tested also positive by culture. CT infection was cleared in 73 women (94\%), of whom 61 (78\%) at their second FU visit (median 21 days; IQR 21-25). Of the five women who did not clear their infection, three were also culture positive indicating a viable infection. All five reported unprotected sexual contact after inclusion prior to their last FU visit, indicating potentially new infections.

Conclusion We observed prolonged and intermittent positive results over time for both NAAT tests. For three participants (4\%) viable CT infections were detected 49 days after treatment. All three cases reported new sexual contacts. In conclusion, persisting infections or treatment failure were rare.

Support: Hologic provided Aptima test materials and kits inkind. Roche provided Cobas test materials and kits in-kind. Copan provided Universal Transport Medium in-kind

\section{P3.19 RISK FACTORS FOR HIV INFECTION AMONG FEMALE COMMERCIAL SEX WORKERS IN BANGUI, CENTRAL AFRICAN REPUBLIC}

${ }^{1}$ Belec, ${ }^{2}$ Jean De Dieu Longo. ${ }^{1}$ Paris V University, Paris, France; ${ }^{2}$ Centre National de Référence Des Maladies Sexuellement Transmissibles Et de La Thérapie Antirétrovir, Bangui - Central African Republic

\subsection{6/sextrans-2017-053264.256}

Introduction The categorization of female commercial sex work according socio-anthropologic criteria constitutes a prerequisite to assess differential risks of HIV exposure.

Methods A cross-sectional questionnaire survey was conducted to describe the spectrum of commercial sex work in Bangui, the capital city of the Central African Republic, among 345 sexually active women having more than 2 sexual partners other than their regular partner during the last 3 months and reporting to have received money or "gifts" in return of their sexual relationships.

Results HIV infection in study female sex worker (FSW) population was strongly associated with anal sex practice with last clients (OR, 4.3), irregular condom use in last 3 months (OR, 24.9), and alcohol consumption before sex (adjusted OR, 2.8). Networks of commercial sex work comprised six different FSW categories, including two groups of "official” professional FSW primarily classified according to their site of work [i) "kata" (18.6\%) representing women working in poor neighbourhoods of Bangui; ii) "pupulenge" (13.9\%) working in hotels and night clubs to seek White men] and four groups of "clandestine" nonprofessional FSW classified according to their reported main activity [i) "market and street vendors" $(20.8 \%)$; ii) "schoolgirls or students" (19.1\%) involved in occasional transactional sex (during holydays); iii) "housewives or unemployed women" (15.7\%); “civil servants" (11.9\%) working as soldiers or in public sector]. HIV varied according to FSW categories. HIV prevalence was 6-fold higher among "kata" than "pupulenge" (39.1\% versus 6.3\%). "Students", "civil servants" and "housewives" were the less HIV-infected (6.1\%, 9.8\%, 13.0\%, respectively), whereas "sellers" constituted the category of highest HIV prevalence (31.9\%).

Conclusion Our observations highlight the high level of vulnerability of both poor professional "kata" and nonprofessional "street vendors" FSW categories which should be particularly taken in account when designing prevention programs for STIs/HIV control purposes.

\section{P3.20 PREVALENCE OF CHLAMYDIA TRACHOMATIS, NEISSERIA GONORRHOEAE AND TRICHOMONAS VAGINALIS IN FEMALE SEX WORKERS IN MOROCCO}

${ }^{1} B$ Bellaji, ${ }^{1} A$ Hançali, ${ }^{1} S$ Jennane, ${ }^{1} \mathrm{H}$ Oukouchoud, ${ }^{1} \mathrm{C}$ Ouanaim, ${ }^{2} \mathrm{~A}$ Bennani, ${ }^{2} \mathrm{~A}$ Latifi, ${ }^{1} \mathrm{R}$ Charof. ${ }^{1}$ National Institute of Hygiene, Rabat, Morocco; ${ }^{2}$ National Aids Program, Ministry of Health, Rabat, Morocco

\subsection{6/sextrans-2017-053264.257}

Introduction The incidence of reported sexually transmitted infections (STIs) in Morocco is over than 350.000 per year. The management of cases is based on syndromic approach especially in women. While female sex workers (FSWs) are assumed to be at increased risk of STIs, there are limited comparative data with other population groups available. Two studies have been conducted between 2013-2014 in different cities in Morocco in order, to explore the prevalence of Chlamydia trachomatis (CT), Neisseria gonorrhoeae (GC), and Trichomonas vaginalis (TV) among FSWs and to compare them with prevalences found in women consultant in family planning units (FPU).

Methods A simple of 519 FSWs and 537 asymptomatic women consultant in FPU was recruited in basic health services and NGOs. All consenting FSWs and women consultant in FPU underwent pretest counselling and provided socio demographic and behavioural data using a structured questionnaire. The women were also asked to provide vaginal and cervical specimens to detect the respective STIs. GC identification was performed by culture and PCR, CT was detected by PCR and TV was detected by culture.

Results The prevalence of CT, GC, and TV were 20,7\%, $9,35 \%$, and $13,3 \%$, respectively, in the FSWs, compared with $3 \%, 0,4 \%$, and $5,6 \%$ respectively in the women consultant in FPU. These results show a high prevalence of CT, GC and TV in Moroccan FSWs than women consultant in FPU.

Conclusion Most STIs prevalences are lower in comparison with prevalences found for FSWs in other countries. However, the National Aids Program conclude that continued close monitoring of the prevalence of CT, GC and TV infection in FSWs is important for preventing the dissemination of these microorganisms, and that further investigation of CT as a sexually transmitted pathogen in women is needed. 